\title{
Study of pathogenic features of stress-related disorders by proteomics methods
}

\author{
A. Seregin ${ }^{1}$, L. Loginova ${ }^{1}$, A. Letova ${ }^{2 *}$, E. Dmitrieva ${ }^{1}$, N. Karageorgiy ${ }^{3}$, \\ V. Rudnitckiy ${ }^{1}$, L. Smirnova ${ }^{1}$ \\ ${ }^{1}$ Mental Health Research Institute, Tomsk National Research Medical Center of the RAS, Tomsk, Russia \\ ${ }^{2}$ Siberian State Medical University, Tomsk, Russia \\ ${ }^{3}$ Tomsk State Pedagogical University, Tomsk, Russia \\ *e-mail: anastasia.a.letova@gmail.com
}

Key words: biomarkers, biological psychiatry, adjustment disorder, protein markers

\begin{abstract}
Motivation and Aim: Stress-related disorders are the group of mental illnesses, combined conditions induced by acute or chronic stress. One of these disorders is the adjustment disorder - the pathological condition of subjective distress and emotional disorder, which make difficulties for social activity in adaptation period to significant changes in life. In the base of the pathogenesis of adjustment, disorder lays a classical theory of stress, but many aspects of its still cannot be explained. Proteomics methods could promote the solution of this problem and answer the question about laboratory diagnostic of some mental disorders.
\end{abstract}

Methods and Algorithms: The base of study is the comparative proteomic analysis of blood serum 10 healthy donors and 10 patients with adjustment disorder.Firstly, the serum was purified from six major proteins by affinity chromatography. Thereupon received protein mix was separated by $1 \mathrm{D}$ electrophoresis. After the sample processing, including trypsinolysis and extraction of the peptides, HPLC/mass-spectrometry LTQ Velos (Thermo Scientific) identified the proteins. The validity of results was checked by the non-parametric Fisher exact test with the Yates correction.

Results: According to the results of mass-spectrometry, the next proteins were obtained: Inter-alpha-trypsin inhibitor complex component III (93402 Da), Glucocorticoid receptor AF-1 specific elongation factor (46240 Da), Secretory actin-binding protein (16562 Da) and Hypothetical protein CAE 93899 (14466 Da). Three proteins are participants in modulating the immune response. According to the literature analysis, the secretory actin-binding protein is probably the main acting link in the function of the immune system $[1,2]$. The function of the hypothetical protein is not established yet; the remaining proteins realize the adaptive response of the organism to stress.

Conclusion: Presented proteins glucocorticoid receptor AF-1 specific elongation factor and actin-binding protein could pretend to the role of adjustment disorder biomarkers, reflecting key features of pathogenesis this illness.

\section{References}

1. Adair J. (2009) Inter- $\alpha$-trypsin Inhibitor Promotes Bronchial Epithelial Repair after Injury through Vitronectin Binding. J Biol Chem. 284(25):16922-16930.

2. Choi-Miura N.H. (2001) Quantitative measurement of the novel human plasma protein, IHRP, by sandwich ELISA. Biological Pharmaceutical Bulletin. 24(3):214-217. 